\title{
Dependency Parsing as a Classification Problem
}

\author{
Deniz Yuret \\ Koç University \\ Istanbul, Turkey \\ dyureteku.edu.tr
}

\begin{abstract}
This paper presents an approach to dependency parsing which can utilize any standard machine learning (classification) algorithm. A decision list learner was used in this work. The training data provided in the form of a treebank is converted to a format in which each instance represents information about one word pair, and the classification indicates the existence, direction, and type of the link between the words of the pair. Several distinct models are built to identify the links between word pairs at different distances. These models are applied sequentially to give the dependency parse of a sentence, favoring shorter links. An analysis of the errors, attribute selection, and comparison of different languages is presented.
\end{abstract}

\section{Introduction}

This paper presents an approach to supervised learning of dependency relations in a language using standard machine learning techniques. The treebanks (Hajič et al., 2004; Chen et al., 2003; Böhmová et al., 2003; Kromann, 2003; van der Beek et al., 2002; Brants et al., 2002; Kawata and Bartels, 2000; Afonso et al., 2002; Džeroski et al., 2006; Civit Torruella and Martí Antonín, 2002; Nilsson et al., 2005; Oflazer et al., 2003; Atalay et al., 2003) provided for the CoNLL shared task(Buchholz et al., 2006) were converted to a set of instances each of which consists of the attributes of a candidate word pair with a classification that indicates the existence, direction and type of the dependency link between the pair.

An initial model is built to identify dependency relations between adjacent word pairs using a decision list learning algorithm. To identify longer distance relations, the adjacent modifiers are dropped from the sentence and a second order model is built based on the word pairs that come into contact. A total of three models were built using this technique successively and used for parsing.

All given attributes are considered as candidates in an attribute selection process before building each model. In addition, attributes indicating suffixes of various lengths and character type information were constructed and used.

To parse a given sentence, the models are applied sequentially, each one considering candidate word pairs and adding new links without deleting the existing links or creating conflicts (cycles or crossings) with them. Thus, the algorithm can be considered a bottom-up, multi-pass, deterministic parser. Given a candidate word pair, the models may output "no link", or give a link with a specified direction and type. Thus labeling is an integrated step. Word pair candidates that may form cycles or crossings are never considered, so the parser will only generate projective structures.

Section 2 gives the details of the learning algorithm. Section 3 describes the first pass model of links between adjacent words. Section 4 details the approach for identifying long distance links and presents the parsing results. 


\section{The Learning Algorithm}

The Greedy Prepend Algorithm (Yuret and Ture, 2006) was used to build decision lists to identify dependency relations. A decision list is an ordered list of rules where each rule consists of a pattern and a classification (Rivest, 1987). The first rule whose pattern matches a given instance is used for its classification. In our application the pattern specifies the attributes of the two words to be linked such as parts of speech and morphological features. The classification indicates the existence and the type of the dependency link between the two words.

Table 1 gives a subset of the decision list that identifies links between adjacent words in German. The class column indicates the type of the link, the pattern contains attributes of the two candidate words $\mathrm{X}$ and Y, as well as their neighbors (XL1 indicates the left neighbor of $\mathrm{X}$ ). For example, given the part of speech sequence APPR-ART-NN, there would be an NK link between APPR and ART (matches rule 3), but there would be no link between ART and NN (rule 1 overrides rule 2 ).

\begin{tabular}{lll}
\hline Rule & Class & Pattern \\
\hline 1 & NONE & XL1:postag=APPR \\
2 & L:NK & X:postag=ART Y:postag=NN \\
3 & R:NK & X:postag=APPR \\
4 & NONE & \\
\hline
\end{tabular}

Table 1: A four rule decision list for adjacent word dependencies in German

The average training instance for the dependency problem has over 40 attributes describing the two candidate words including suffixes of different lengths, parts of speech and information on neighboring words. Most of this information may be redundant or irrelevant to the problem at hand. The number of distinct attribute values is on the order of the number of distinct word-forms in the training set. GPA was picked for this problem because it has proven to be fairly efficient and robust in the presence of irrelevant or redundant attributes in previous work such as morphological disambiguation in Turkish (Yuret and Ture, 2006) and protein secondary structure prediction (Kurt, 2005).

\section{Dependency of Adjacent Words}

We start by looking at adjacent words and try to predict whether they are linked, and if they are, what type of link they have. This is a nice subproblem to study because: (i) It is easily converted to a standard machine learning problem, thus amenable to common machine learning techniques and analysis, (ii) It demonstrates the differences between languages and the impact of various attributes. The machine learning algorithm used was GPA (See Section 2) which builds decision lists.

Table 2 shows the percentage of adjacent tokens that are linked in the training sets for the languages studied $^{1}$. Most languages have approximately half of the adjacent words linked. German, with $42.15 \%$ is at the low end whereas Arabic and Turkish with above $60 \%$ are at the high end. The differences may be due to linguistic factors such as the ubiquity of function words which prefer short distance links, or it may be an accident of data representation: for example each token in the Turkish data represents an inflectional group, not a whole word.

\begin{tabular}{llll}
\hline Arabic & 61.02 & Japanese & 54.81 \\
Chinese & 56.59 & Portuguese & 50.81 \\
Czech & 48.73 & Slovene & 45.62 \\
Danish & 55.93 & Spanish & 51.28 \\
Dutch & 55.54 & Swedish & 48.26 \\
German & 42.15 & Turkish & 62.60 \\
\hline
\end{tabular}

Table 2: Percentage of adjacent tokens linked.

\subsection{Attributes}

The five attributes provided for each word in the treebanks were the wordform, the lemma, the coarse-grained and fine-grained parts of speech, and a list of syntactic and/or morphological features. In addition I generated two more attributes for each word: suffixes of up to $n$ characters (indicated by $\operatorname{suffix}[\mathrm{n}])$, and character type information, i.e. whether the word contains any punctuation characters, upper case letters, digits, etc.

Two questions to be answered empirically are: (i) How much context to include in the description of each instance, and (ii) Which attributes to use for each language.

\footnotetext{
${ }^{1}$ Including non-scoring tokens
} 
Table 3 shows the impact of using varying amounts of context in Spanish. I used approximately 10,000 instances for training and 10,000 instances for testing. Only the postag feature is used for each word in this experiment. As an example, consider the word sequence $w_{1} \ldots w_{i} w_{i+1} \ldots w_{n}$, and the two words to be linked are $w_{i}$ and $w_{i+1}$. Context $=0$ means only information about $w_{i}$ and $w_{i+1}$ is included, context $=1$ means we also include $w_{i-1}$ and $w_{i+2}$, etc. The table also includes the number of rules in each decision list. The results are typical of the experiments performed with other languages and other attribute combinations: there is a statistically significant improvement going from context $=0$ to context=1. Increasing the context size further does not have a significant effect.

\begin{tabular}{lll}
\hline Context & Rules & Accuracy \\
\hline 0 & 161 & 83.17 \\
1 & 254 & 87.31 \\
2 & 264 & 87.05 \\
3 & 137 & 87.14 \\
\hline
\end{tabular}

Table 3: Context size vs. accuracy in Spanish.

A number of experiments were run to determine the best attribute combinations for each language. Table 4 gives a set of results for single attributes in Spanish. These results are based on 10,000 training instances and all experiments use context $=1$. Postag was naturally the most informative single attribute on all languages tested, however the second best or the best combination varied between languages. Suffix[3] indicates all suffixes up to three characters in length. The FEATs column was split into its constituent features each of which was treated as a binary attribute.

\begin{tabular}{lll}
\hline Attributes & Rules & Accuracy \\
\hline postag & 254 & 87.31 \\
cpostag & 154 & 85.72 \\
suffix[3] & 328 & 77.15 \\
lemma & 394 & 76.78 \\
form & 621 & 75.06 \\
feats & 66 & 71.95 \\
ctype & 47 & 53.40 \\
\hline
\end{tabular}

Table 4: Attributes vs. accuracy in Spanish.
There are various reasons for performing attribute selection. Intuitively, including more information should be good, so why not use all the attributes? First, not every machine learning algorithm is equally tolerant of redundant or irrelevant attributes. Naive Bayes gets $81.54 \%$ and $\mathrm{C} 4.5$ gets $86.32 \%$ on the Spanish data with the single postag attribute using context=1. One reason I chose GPA was its relative tolerance to redundant or irrelevant attributes. However, no matter how robust the algorithm, the lack of sufficient training data will pose a problem: it becomes difficult to distinguish informative attributes from non-informative ones if the data is sparse. About half of the languages in this study had less than 100,000 words of training data. Finally, studying the contribution of each attribute type in each language is an interesting research topic in its own right. The next section will present the best attribute combinations and the resulting accuracy for each language.

\subsection{Results}

\begin{tabular}{lll}
\hline Language & Attributes & Accuracy \\
\hline Arabic & ALL & 76.87 \\
Chinese & postag, cpostag & 84.51 \\
Czech & postag, lemma & 79.25 \\
Danish & postag, form & 86.96 \\
Dutch & postag, feats & 85.36 \\
German & postag, form & 87.97 \\
Japanese & postag, suffix[2] & 95.56 \\
Portuguese & postag, lemma & 90.18 \\
Slovene & ALL & 85.19 \\
Spanish & postag, lemma & 89.01 \\
Swedish & postag, form & 83.20 \\
Turkish & ALL & 85.27 \\
\hline
\end{tabular}

Table 5: Adjacent word link accuracy.

Table 5 gives the best attribute combinations for determining adjacent word links for each language studied. The attribute combinations and the corresponding models were determined using the training sets, and the accuracy reported is on the test sets. These attribute combinations were used as part of the model in the final evaluation. I used context=1 for all the models. Because of time limitations attribute combinations with more than two attributes 
could not be tested and only the first 100,000 training instances were used. Exceptions are indicated with "ALL", where all attributes were used in the model - these are cases where using all the attributes outperformed other subsets tried.

For most languages, the adjacent word link accuracy is in the $85-90 \%$ range. The outliers are Arabic and Czech at the lower end, and Japanese at the higher end. It is difficult to pinpoint the exact reasons: Japanese has the smallest set of link types, and Arabic has the greatest percentage of adjacent word links. Some of the differences between the languages come from linguistic origins, but many are due to the idiosyncrasies of our particular data set: number of parts of speech, types of links, quality of the treebank, amount of data are all arbitrary factors that effect the results. One observation is that the ranking of the languages in Table 5 according to performance is close to the ranking of the best results in the CoNLL shared task - the task of linking adjacent words via machine learning seems to be a good indicator of the difficulty of the full parsing problem.

\section{Long Distance Dependencies}

Roughly half of the dependency links are between non-adjacent words in a sentence. To illustrate how we can extend the previous section's approach to long distance links, consider the phrase "kick the red ball". The adjacent word linker can only find the red-ball link even if it is $100 \%$ accurate. However once that link has been correctly identified, we can drop the modifier "red" and do a second pass with the words "kick the ball". This will identify the link the-ball, and dropping the modifier again leaves us with "kick ball". Thus, doing three passes over this word sequence will bring all linked words into contact and allow us to use our adjacent word linker. Table 6 gives the percentage of the links discovered in each pass by a perfect model in Spanish.

\begin{tabular}{lrrrrr}
\hline Pass: & 1 & 2 & 3 & 4 & 5 \\
Link\%: & 51.09 & 23.56 & 10.45 & 5.99 & 3.65 \\
\hline
\end{tabular}

Table 6: Spanish links discovered in multiple passes.

We need to elaborate a bit on the operation of "dropping the modifiers" that lead from one pass to the next. After the discovery of the red-ball link in the above example, it is true that "red" can no longer link with any other words to the right (it cannot cross its own head), but it can certainly link with the words to the left. To be safe, in the next pass we should consider both the-red and the-ball as candidate links. In the actual implementation, given a partial linkage, all "potentially adjacent" word pairs that do not create cycles or link crossings were considered as candidate pairs for the next pass.

There are significant differences between the first pass and the second pass. Some word pairs will rarely be seen in contact during the first pass (e.g. "kick ball"). Maybe more importantly, we will have additional "syntactic" context during the second pass, i.e. information about the modifiers discovered in the first pass. All this argues for building a separate model for the second pass, and maybe for further passes as well.

In the actual implementation, models for three passes were built for each language. To create the training data for the n'th pass, all the links that can be discovered with (n-1) passes are taken as given, and all word pairs that are "potentially adjacent" given this partial linkage are used as training instances. To describe each training instance, I used the attributes of the two candidate words, their surface neighbors (i.e. the words they are adjacent to in the actual sentence), and their syntactic neighbors (i.e. the words they have linked with so far).

To parse a sentence the three passes were run sequentially, with the whole sequence repeated twice ${ }^{2}$. Each pass adds new links to the existing partial linkage, but does not remove any existing links. Table 7 gives the labeled and unlabeled attachment score for the test set of each language using this scheme.

\section{Conclusion}

I used standard machine learning techniques to investigate the lower bound accuracy and the impact of various attributes on the subproblem of identifying dependency links between adjacent words. The technique was then extended to identify long distance dependencies and used as a parser. The model gives average results for Turkish and Japanese but

\footnotetext{
${ }^{2}$ This counterintuitive procedure was used because it gave the best results on the training set.
} 


\begin{tabular}{lll}
\hline Language & LAS & UAS \\
\hline Arabic & 52.42 & 68.82 \\
Chinese & 72.72 & 78.37 \\
Czech & 51.86 & 66.36 \\
Danish & 71.56 & 78.16 \\
Dutch & 62.75 & 66.17 \\
German & 63.82 & 67.71 \\
Japanese & 84.35 & 87.31 \\
Portuguese & 70.35 & 79.46 \\
Slovene & 55.06 & 70.60 \\
Spanish & 69.63 & 73.89 \\
Swedish & 65.23 & 73.25 \\
Turkish & 60.31 & 71.54 \\
\hline
\end{tabular}

Table 7: Labeled and unlabeled attachment scores.

generally performs below average. The lack of a specialized parsing algorithm taking into account sentence wide constraints and the lack of a probabilistic component in the model are probably to blame. Nevertheless, the particular decomposition of the problem and the simplicity of the resulting models provide some insight into the difficulties associated with individual languages.

\section{References}

A. Abeillé, editor. 2003. Treebanks: Building and Using Parsed Corpora, volume 20 of Text, Speech and Language Technology. Kluwer Academic Publishers, Dordrecht.

S. Afonso, E. Bick, R. Haber, and D. Santos. 2002. "Floresta sintá(c)tica": a treebank for Portuguese. In Proc. of the Third Intern. Conf. on Language Resources and Evaluation (LREC), pages 1698-1703.

N. B. Atalay, K. Oflazer, and B. Say. 2003. The annotation process in the Turkish treebank. In Proc. of the 4th Intern. Workshop on Linguistically Interpreteted Corpora (LINC).

A. Böhmová, J. Hajič, E. Hajičová, and B. Hladká. 2003. The PDT: a 3-level annotation scenario. In Abeillé (Abeillé, 2003), chapter 7.

S. Brants, S. Dipper, S. Hansen, W. Lezius, and G. Smith. 2002. The TIGER treebank. In Proc. of the First Workshop on Treebanks and Linguistic Theories (TLT).

S. Buchholz, E. Marsi, A. Dubey, and Y. Krymolowski. 2006. CoNLL-X shared task on multilingual dependency parsing. In Proc. of the Tenth Conf. on Com- putational Natural Language Learning (CoNLL-X). SIGNLL.

K. Chen, C. Luo, M. Chang, F. Chen, C. Chen, C. Huang, and Z. Gao. 2003. Sinica treebank: Design criteria, representational issues and implementation. In Abeillé (Abeillé, 2003), chapter 13, pages 231-248.

M. Civit Torruella and M ${ }^{\mathrm{a}}$ A. Martí Antonín. 2002. Design principles for a Spanish treebank. In Proc. of the First Workshop on Treebanks and Linguistic Theories (TLT).

S. Džeroski, T. Erjavec, N. Ledinek, P. Pajas, Z. Žabokrtsky, and A. Žele. 2006. Towards a Slovene dependency treebank. In Proc. of the Fifth Intern. Conf. on Language Resources and Evaluation (LREC).

J. Hajič, O. Smrž, P. Zemánek, J. Šnaidauf, and E. Beška. 2004. Prague Arabic dependency treebank: Development in data and tools. In Proc. of the NEMLAR Intern. Conf. on Arabic Language Resources and Tools, pages $110-117$.

Y. Kawata and J. Bartels. 2000. Stylebook for the Japanese treebank in VERBMOBIL. VerbmobilReport 240, Seminar für Sprachwissenschaft, Universität Tübingen.

M. T. Kromann. 2003. The Danish dependency treebank and the underlying linguistic theory. In Proc. of the Second Workshop on Treebanks and Linguistic Theories $(T L T)$.

Volkan Kurt. 2005. Protein structure prediction using decision lists. Master's thesis, Koç University.

J. Nilsson, J. Hall, and J. Nivre. 2005. MAMBA meets TIGER: Reconstructing a Swedish treebank from antiquity. In Proc. of the NODALIDA Special Session on Treebanks.

K. Oflazer, B. Say, D. Zeynep Hakkani-Tür, and G. Tür. 2003. Building a Turkish treebank. In Abeillé (Abeillé, 2003), chapter 15.

Ronald L. Rivest. 1987. Learning decision lists. $M a-$ chine Learning, 2:229-246.

L. van der Beek, G. Bouma, R. Malouf, and G. van Noord. 2002. The Alpino dependency treebank. In Computational Linguistics in the Netherlands (CLIN).

Deniz Yuret and Ferhan Ture. 2006. Learning morphological disambiguation rules for Turkish. In HLTNAACL 06. 